\title{
Early experience with chemotherapy intensification for poor prognosis metastatic germ cell cancer and unfavorable tumor marker decline
}

\author{
Anupam Batra ${ }^{1}$; Scott Ernst ${ }^{1}$; Kylea Potvin ${ }^{1}$; Ricardo Fernandes ${ }^{1}$; Nicholas Power ${ }^{2}$; James \\ Vanhie $^{3}$; Eric Winquist ${ }^{1}$ \\ ${ }^{1}$ Division of Medical Oncology, Department of Oncology, Western University and London Health Sciences Centre, \\ London, ON, Canada; ${ }^{2}$ Division of Urology, Department of Surgery, Western University and London Health \\ Sciences Centre, London, ON, Canada; ${ }^{3}$ London Health Sciences Centre, London, ON, Canada
}

Cite as: Can Urol Assoc J 2019 July 23; Epub ahead of print. http://dx.doi.org/10.5489/cuaj.5802

Published online July 23, 2019

$* * *$

\section{Abstract}

Introduction: Intensified chemotherapy improved outcomes for men with poor prognosis metastatic germ cell cancer (GCC) and unfavorable tumor marker decline (UTMD) after one cycle of bleomycin, etoposide, and cisplatin (BEP) chemotherapy in the GETUG-13 trial. Herein, we report our experience to date using a similar approach.

Methods: Patients were identified from our electronic GCC database. Men with poor prognosis GCC and UTMD were offered intensified chemotherapy consisting of T-BEP (three cycles) plus paclitaxel, ifosfamide, and cisplatin (TIP) (one cycle), along with prophylactic granulocytecolony stimulating factor (G-CSF) and resection of residual masses. Cisplatin, etoposide and ifosfamide (PEI) replaced last cycle of T-BEP for bleomycin pulmonary concerns. Serious toxicities, progression-free survival, and overall survival were evaluated retrospectively. Results: Ten patients with poor prognosis GCC were identified May 2012 to April 2016. Eight patients had UTMD. Six were offered and received intensified chemotherapy (two T-BEPx3 + TIP and four T-BEPx2 + PEI + TIP). Serious toxicities included neutropenic sepsis, deep venous thrombosis, and $C$. difficile colitis, but there were no toxic deaths. One patient died of synchronous metastatic adenocarcinoma ex teratoma. The remaining five patients achieved marker negative partial response, two had residual mature teratoma excised, and four have no evidence of disease after surgery. All are alive at a median of 57.6 months (range 37.2-65.3 months); one patient has grade 2 peripheral sensory neuropathy, and one patient has grade 2 cognitive disturbance. Of four patients treated with standard BEP, two have died of disease and two are alive at 44.9 and 47.2 months. 
Conclusions: Our experience with intensified chemotherapy for men with poor prognosis GCC and UTMD confirms that it is feasible, reasonably safe, and appears to provide results similar to those reported in GETUG-13.

\section{Introduction}

Men with metastatic germ cell cancer (GCC) are classified for treatment according to the International Germ Cell Consensus Classification (IGCCC) prognostic classification ${ }^{1}$. Of men with newly diagnosed non-seminomatous GCC, 56\% have good prognosis disease, $28 \%$ have intermediate prognosis disease and $16 \%$ have poor prognosis disease; and 5-year survivals are $92 \%, 80 \%$ and 50\%, respectively. Four cycles of either bleomycin, etoposide and cisplatin (BEP) or etoposide, ifosfamide, and cisplatin (VIP) chemotherapy remain the standard treatment for patients with poor prognosis $\mathrm{GCC}^{2,3}$. Considerable effort has been devoted to improving upon the results of BEP in these patients by adding new agents or dose intensification including highdose chemotherapy (HDCT) with stem-cell support ${ }^{4}$. However, randomized trials studying these approaches in unselected patients over the past 25 years have been negative ${ }^{5}$.

In 2004, Fizazi and colleagues reported that patients with poor prognosis GCC and favorable tumor marker decline 3 weeks after the first cycle of BEP had better 4-year progression-free (64\% vs $38 \%$ ) and overall ( $83 \%$ vs $58 \%$ ) survival compared to patients with unfavorable marker decline 6 . These data provided the rationale for the only positive randomized trial in poor prognosis GCC reported to date. GETUG-13 assessed the efficacy of intensified chemotherapy in men with poor prognosis GCC and unfavorable tumor marker decline after one cycle of standard $\mathrm{BEP}^{7}$. Men were randomly assigned to receive either three more cycles of BEP or an intensified chemotherapy regimen consisting of intravenous paclitaxel-BEP (T-BEP) for 2 cycles followed by cisplatin, infusional bleomycin, and ifosfamide (PBI) for 2 cycles, along with doses of oxaliplatin given on day 10 of each cycle. Primary prophylaxis with G-CSF was given each cycle. 254 patients were enrolled and evaluable for TMD, and 203 (80\%) had unfavorable tumor marker decline and were randomized. The results validated first cycle tumor marker decline as a prognostic factor in this population, and provided proof-of-principle for the benefits of chemotherapy dose intensification: 3-year progression-free (59\% versus $48 \%$; hazard ratio [HR] $0.66,95 \% \mathrm{CI} 0 \cdot 44-1 \cdot 00, \mathrm{p}=0 \cdot 05)$ and overall survival ( $73 \%$ versus $65 \%$; HR $0.78,95 \% \mathrm{CI}$ $0.46-1.31, \mathrm{p}=0.34$ ) were improved, and the need for subsequent salvage HDCT was reduced with intensified chemotherapy compared to the BEP control arm (6\% versus 16\%). As expected, more frequent neurological and hematological toxicity was seen with intensified chemotherapy but there was no increase in toxic death.

Although the majority of expert clinicians agree it should be offered to selected patients, consensus is incomplete about use of intensified chemotherapy in this population and it has not been widely adopted ${ }^{2}$. The main points of controversy relate to the components and schedule of the intensified chemotherapy regimen used in GETUG-13 which consisted of a total of four 
additional 21 day cycles of chemotherapy following cycle 1 BEP: cycles 2 and 3 were T-BEP-O (BEP plus paclitaxel $175 \mathrm{mg} / \mathrm{m}^{2}$ IV over 3 hours day 1 and oxaliplatin $130 \mathrm{mg} / \mathrm{m}^{2} \mathrm{IV}$ day 10), and cycles 4 and 5 were PBI (cisplatin $100 \mathrm{mg} / \mathrm{m}^{2}$ IV over 2 hours day 1, bleomycin 25 units daily by continuous IV infusion over 24 hours for 5 days day 10-14, and ifosfamide $2 \mathrm{mg} / \mathrm{m}^{2} \mathrm{IV}$ over 3 hours days 10,12, and 14). Oxaliplatin had initially been included in cycles 4 and 5; however, it was omitted after 12 patients were treated due to excessive peripheral neuropathy, so 89\% (93/105) patients received PBI alone.

A first concern is that this regimen was not developed using a conventional phase I-II approach, and required post hoc modification for safety. Second, although the rationale for addition of oxaliplatin as a first-line agent is apparent from its activity in the palliative treatment of refractory GCC, there is not evidence that adding it to first-line cisplatin-based therapy is beneficial $^{8}$. This is not the case for paclitaxel which appeared to improve outcomes when added to $\mathrm{BEP}^{9}$. Third are safety concerns giving bleomycin beyond 360 units, along with recent evidence that continuous infusion does not reduce pulmonary toxicity, and little evidence for incremental benefit with greater cumulative bleomycin dose ${ }^{10}$. Finally, the rationale for the unconventional administration schedules of cisplatin and ifosfamide in cycles 4 and 5 was unclear, and cisplatin given as a single high dose likely increases toxicity.

In view of these concerns and in the absence of regulatory or funding approval for oxaliplatin for this indication, we began offering chemotherapy intensification with a modified regimen based on the GETUG-13 schedule (see Methods) to men with poor prognosis GCC and unfavorable tumor marker decline at our institution and report our results to date.

\section{Methods}

Eligible men with IGCCC poor prognosis GCC were treated at London Health Sciences Centre between May 2012 and April 2016. Patients were identified from our electronic GCC patient database and had evidence of testicular, retroperitoneal, or mediastinal GCC. Patients with poor prognosis disease had very elevated tumor markers (serum alfa-fetoprotein (AFP) greater than $10,000 \mu \mathrm{g} / \mathrm{L}$, serum human chorionic gonadotropin (HCG) greater than 50,000 IU/L, or serum lactate dehydrogenase (LDH) greater than 10 times the upper limit of the normal range), primary mediastinal non-seminoma, or non-pulmonary visceral metastases. Predicted time to normalization of tumor markers was calculated from baseline and day 22 AFP and/or HCG values as previously described ${ }^{6}$. Patients with a rise in tumor marker at day 22 or time to normalization of tumor markers greater than 9 weeks for AFP or 6 weeks for HCG after cycle 1 BEP or VIP were classified as having unfavorable tumor marker decline and offered chemotherapy intensification starting at cycle 2.

Intensified chemotherapy consisted of T-BEP for cycles 2, 3 and 4 followed by one cycle of TIP (paclitaxel $250 \mathrm{mg} / \mathrm{m}^{2}$ IV over 24 hours day 1, ifosfamide $1500 \mathrm{mg} / \mathrm{m}^{2}$ IV day 2-5 with Mesna, and cisplatin $25 \mathrm{mg} / \mathrm{m}^{2} \mathrm{IV}$ day 2-5), ${ }^{9,11}$. Except for omission of oxaliplatin, the first 2 cycles were identical to the GETUG-13 approach. In GETUG-13, bleomycin dose was reduced 
by at least $20 \%$ in $38 \%$ and $45 \%$ of patients in cycles 4 and 5 , respectively ${ }^{7}$. With omission of oxaliplatin from these cycles and the questionable efficacy of the infusional bleomycin, we were concerned that cycles 4 and 5 provided little more than the equivalent of cisplatin-ifosfamide doublets. We intensified these cycles by using an additional cycle of T-BEP plus TIP, which has a known safety profile and proven effectiveness. This increased treatment intensity in cycles 4 and 5 to compensate for omission of oxaliplatin in cycles 2 and 3 (Table 1). Patients with clinical signs of bleomycin pulmonary toxicity or DLCO/VA less than 75\% after 2 cycles of T-BEP received PEI (cisplatin $20 \mathrm{mg} / \mathrm{m}^{2}$, etoposide $100 \mathrm{mg} / \mathrm{m}^{2}$, and ifosfamide $1.2 \mathrm{gm} / \mathrm{m}^{2}$ all IV days 15 ) instead of the third cycle of T-BEP ${ }^{12}$. Patients received prophylactic G-CSF with all cycles and Mesna was given following ifosfamide.

Tumor markers and chest radiograph were obtained every 3 weeks. Lung function was assessed by spirometry and CO diffusion at baseline and before cycle 4. Restaging whole body CT scan was done after completion of systemic therapy. Patients with residual masses more than $1 \mathrm{~cm}$ were assessed for surgical resection. Patients were followed after treatment once every 2 months for two years, once every 4 months in the third year, once every 6 months in the fourth year, and once every year after the fourth year. Surveillance included clinical examination, tumor markers, and CT scan of the initially involved sites (every 4 months for 2 years, and then annually for the next 3 years). Outcome data were extracted retrospectively. Grade 3-5 toxicities were identified and graded using CTCAE version 4.03. Progression-free and overall survival was calculated from the date of initiation of chemotherapy.

\section{Results}

Between May 01, 2012 and April 30, 2016, 10 men with poor prognosis GCC were identified: 4 had liver metastases, 1 had brain metastases, 2 had bone metastases, and 1 had a small bowel metastasis (Table 2). Eight had unfavorable tumor marker decline: 7 had inadequate drop in AFP or HCG, and one had a rise in AFP, after their first BEP or VIP cycle. Six of these men were offered and received intensified chemotherapy; two were treated with standard BEP. Of the six patients treated with intensified chemotherapy, two received cycles 2-4 T-BEP plus cycle 5 TIP, and four received cycle 2-3 T-BEP, cycle 4 PEI and cycle 5 TIP due to decline in DLCO/VA of greater than $25 \%$ after cycle 3 . One patient who was diagnosed with synchronous metastatic adenocarcinoma ex teratoma during intensified chemotherapy treatment. Two patients had a favorable tumor marker decline by virtue of normal markers pretreatment and no rise in AFP or HCG after cycle $1 \mathrm{BEP}$, and received four cycles of BEP. Of the 4 patients treated with BEP, one had last cycle BEP replaced with VIP for pulmonary toxicity.

The received dose intensity of the intensified chemotherapy was $100 \%$ of that planned for all drugs. Median time from start of first to start of last intensified chemotherapy cycle was 64.5 days (range, 62-70 days) (expected time: 63 days). One patient with choriocarcinoma who had brain metastases and high volume lung metastases received an initial cycle of EP which was complicated by tumor lysis syndrome and multiorgan failure. No other serious pulmonary 
toxicity was observed (Table 3). After recovery, this patient received one cycle of VIP chemotherapy and then 4 cycles of intensified chemotherapy (total 6 cycles). Three patients experienced an episode of febrile neutropenia despite G-CSF prophylaxis. One patient experienced C. difficile colitis after cycles 2 and 4, one had culture-negative neutropenic proctitis, and one had neutropenic sepsis due to E. coli bacteremia. Two patients experienced deep venous thrombosis requiring anticoagulation.

All five patients with pure GCC treated with intensified chemotherapy achieved marker negative partial response (PR). Four had post-chemotherapy retroperitoneal lymph node dissection (RPLND) along with right hepatic lobectomy and orchiectomy in one patient each. Postoperatively, one patient had ascites due to a pancreatic leak which has since resolved. Pathology showed completely resected teratoma in two patients. Of 4 patients treated with standard BEP, one patient had complete response, two had marker negative PR, and one had marker positive PR. The latter patient had rapid relapse and died of treatment complications during the first cycle of salvage chemotherapy. One patient with marker negative PR had hepatic resection demonstrating residual GCC and died of acute pulmonary embolism before starting salvage treatment.

There were no toxic deaths with intensified chemotherapy. One patient died of synchronously diagnosed metastatic adenocarcinoma ex teratoma after 27 months with peripheral sensory neuropathy requiring treatment with gabapentin. The five pure GCC patients treated with intensified chemotherapy are all alive at a median of 57.6 months (range, 37.2-65.3 months). Four have no evidence of disease (NED) and one patient has residual teratoma being observed in consideration of the risks of repeat surgery. Four have returned to usual school or work, and the remaining patient has been unable to return to work due to grade 2 cognitive dysfunction. One patient who received a total of 6 cycles of chemotherapy, experienced grade 3 peripheral sensory neuropathy now resolved to grade 2 . Of the 4 patients who received standard BEP chemotherapy, two relapsed and died after 5.5 and 7.8 months, and two are NED at a median of 44.9 and 47.2 months.

\section{Discussion}

Although cure is expected for most men with metastatic GCC, poor prognosis disease remains a challenge. In 1997 the IGCCCG reported just a 48\% 5-year overall survival in these patients ${ }^{1}$. Results have improved over the past 20 years due to incremental improvements in care delivery $^{14}$. In the GETUG-13 trial, Fizazi et al ${ }^{7}$ confirmed the prognostic validity of first cycle tumor marker decline and a benefit of intensified chemotherapy in patients with an unfavorable decline. 3-year overall survival in patients treated with standard BEP chemotherapy was 84\% in patients with favorable tumor marker decline, and 65\% in those with unfavorable decline. This confirms that results have improved but, as $80 \%$ of patients had an unfavorable tumor marker decline, there is still need further improvement in treatment. Fortunately, poor prognosis GCC is uncommon, but this also makes it difficult to study. We identified only 10 patients with poor 
prognosis GCC at our referral centre over a 4 year period, and 80\% had unfavorable tumor marker decline, identical to GETUG-13.

Despite the results of GETUG-13, intensified chemotherapy for patients with unfavorable tumor marker decline has not been widely adopted in North America. The reasons for this are not entirely clear, but concerns about the rationale, safety, and availability of drugs included in the intensified chemotherapy regimen used are likely. As oxaliplatin for curative GCC treatment remains unavailable in our jurisdiction, we modified the GETUG-13 intensified chemotherapy approach using standardized regimens familiar to medical oncologists treating GCC. T-BEP was developed by the EORTC using a conventional drug development approach and was tested showing favorable results in intermediate prognosis GCC in a phase III trial ${ }^{9,13}$. Compared to the GETUG-13 approach, our approach did not use oxaliplatin but provided an identical cisplatin dose, 50\% higher etoposide dose, 121\% higher paclitaxel dose, and 50\% lower ifosfamide dose. In patients receiving cycle $4 \mathrm{PEI}$, cisplatin and ifosfamide doses were identical, etoposide was $50 \%$ higher, and paclitaxel 71\% higher. These regimens were delivered at full dose and on schedule. Although open to critique, we propose that the increased etoposide and paclitaxel doses given with our approach more than adequately compensate for the omission of two oxaliplatin doses and reduced total bleomycin dose compared to GETUG-13.

Although intensified chemotherapy was reasonably well tolerated, the risks should not be trivialized. Serious complications occurred during treatment that included neutropenic sepsis despite G-CSF, C. difficile colitis, and deep venous thrombosis. More aggressive use of G-CSF prophylaxis may be warranted with our approach. The assessment and management of bleomycin pulmonary toxicity remains controversial. Shamash and colleagues ${ }^{10}$ have suggested that routine pulmonary function testing is of little value, and that development of cough and findings on chest CT are better identifiers. We tended to be conservative, and four of six patients had PEI substituted for cycle 3 T-BEP due to decline in DLCO/VA of greater than 25\%. Our intended bleomycin doses were no more than $34 \%$ and $56 \%$ less the doses actually received by patients in the GETUG-13 trial. Including cycle 1 BEP bleomycin, 4 patients received a total dose of 270 units of bleomycin, and one each received 240 units and 360 units. Two patients experienced grade 3 peripheral sensory neuropathy that resolved to grade 2 in one patient. One patient has not returned to usual work due to grade 2 cognitive disturbance and grade 1 dizziness. Increased paclitaxel exposure could contribute to both neuropathy and pulmonary toxicity.

Our report is limited by its small sample size, nevertheless, all five pure GCC patients treated with intensified chemotherapy remain alive at a median follow up of nearly 5 years which appears to be compatible with GETUG-13 (75\% overall survival at five years), and no patients have required salvage high-dose chemotherapy. We demonstrate that intensified chemotherapy based on the GETUG-13 approach in patients with unfavorable tumor decline after one cycle of BEP chemotherapy is feasible in a Canadian setting. However, the use of intensified chemotherapy should be restricted to high-volume GCC centres, consistent with recent expert recommendations that poor prognostic GCC patients be managed in this environment ${ }^{14}$. 


\section{Conclusions}

Our experience to date suggests that use of intensified chemotherapy for men with poor prognosis metastatic germ cell cancer and unfavorable tumor marker decline after one cycle of BEP is feasible and reasonably safe. Our outcomes appear to be similar to those reported in GETUG-13. Although GETUG-13 provided proof-of-principle for chemotherapy intensification, the optimal intensified chemotherapy regimen remains controversial. In our approach we optimizing doses of etoposide and paclitaxel using standard combination regimens to compensate for omission of oxaliplatin, which was unavailable, and reduced exposure to bleomycin. Peripheral sensory neuropathy was the most common long term adverse effect noted, likely due to cumulative exposure to cisplatin and paclitaxel. Notwithstanding limited patient numbers, this report supports further investigation of dose intensification in treating high risk poor prognosis metastatic testicular cancer patients. 


\section{References}

1. International Germ Cell Cancer Collaborative Group. International germ cell consensus classification: a prognostic factor-based staging system for metastatic germ cell cancers. $J$ Clin Oncol 1997 15:594-603.

2. Honecker F, Aparicio J, Berney D, et al. ESMO Consensus Conference on testicular germ cell cancer: diagnosis, treatment and follow-up. Ann Oncol 2018 29:1658-86.

3. Nichols CR, Catalano PJ, Crawford ED, et al. Randomized comparison of cisplatin and etoposide and either bleomycin or ifosfamide in treatment of advanced disseminated germ cell tumors: An Eastern Cooperative Oncology Group, Southwest Oncology Group, and Cancer and Leukemia Group B Study. J Clin Oncol 1998 16:1287-93.

4. Motzer RJ, Nichols CJ, Margolin KA, et al. Phase III randomized trial of conventionaldose chemotherapy with or without high-dose chemotherapy and autologous hematopoietic stem-cell rescue as first-line treatment for patients with poor-prognosis metastatic germ cell tumors. J Clin Oncol 2007 25:247-56.

5. Calabrò F, Albers $\mathrm{P}$, Bokemeyer $\mathrm{C}$, et al. The contemporary role of chemotherapy for advanced testis cancer: a systematic review of the literature. Eur Urol 2012 61:1212-21.

6. Fizazi K, Culine S, Kramar A, et al. Early predicted time to normalization of tumor markers predicts outcome in poor-prognosis nonseminomatous germ cell tumors. J Clin Oncol 2004 22:3868-76.

7. Fizazi K, Pagliaro L, Laplanche A, et al. Personalised chemotherapy based on tumour marker decline in poor prognosis germ-cell tumours (GETUG-13): a phase 3, multicenter, randomised trial. Lancet Oncol 2014 15:1442-50.

8. Oechsle K, Kollmannsberger C, Honecker F, et al; German Testicular Cancer Study Group. Long-term survival after treatment with gemcitabine and oxaliplatin with and without paclitaxel plus secondary surgery in patients with cisplatin-refractory and/or multiply relapsed germ cell tumors. Eur Urol 2011 60:850-5.

9. de Wit R, Skoneczna I, Daugaard G, et al. Randomized phase III study comparing paclitaxel-bleomycin, etoposide, and cisplatin (BEP) to standard BEP in intermediateprognosis germ-cell cancer: Intergroup study EORTC 30983. J Clin Oncol 2012 30:7929.

10. Shamash J, Sarker SJ, Huddart R, et al. A randomized phase III study of $72 \mathrm{~h}$ infusional versus bolus bleomycin in BEP (bleomycin, etoposide and cisplatin) chemotherapy to treat IGCCCG good prognosis metastatic germ cell tumours (TE-3). Ann Oncol 2017 1;28:1333-8

11. Motzer RJ, Sheinfeld J, Mazumdar M, et al. Paclitaxel, ifosfamide, and cisplatin secondline therapy for patients with relapsed testicular germ cell cancer. J Clin Oncol 2000 18:2413-8.

12. Harstrick A, Schmoll HJ, Bokemeyer C, et al. Cisplatin/etoposide/ifosfamide stepwise dose escalation with concomitant granulocyte/macrophage-colony-stimulating factor for patients with far-advanced testicular carcinoma. J Cancer Res Clin Oncol 1991117 Suppl 4:S198-202.

13. de Wit R, Louwerens M, de Mulder PH, et al. Management of intermediate-prognosis germ-cell cancer: results of a phase I/II study of Taxol-BEP. Int J Cancer 1999 83:831-3. 
14. Tandstad T, Kollmannsberger CK, Roth BJ, et al. Practice makes perfect: the rest of the story in testicular cancer as a model curable neoplasm. J Clin Oncol 2017 35:3525-8. 
Figures and Tables

Table 1. Chemotherapy dosing schedule

\begin{tabular}{|c|c|c|c|c|c|c|c|c|}
\hline & \multicolumn{2}{|c|}{ GETUG-13 } & \multicolumn{3}{|c|}{ T-BEP/TIP } & \multicolumn{3}{|c|}{ T-BEP/PEI/TIP } \\
\hline Drug & $\begin{array}{c}\text { Total } \\
\text { dose } \\
\left(\mathrm{mg} / \mathbf{m}^{2}\right)\end{array}$ & $\begin{array}{c}\text { Dose } \\
\text { intensity } \\
\text { (dose/week) }\end{array}$ & $\begin{array}{c}\text { Total } \\
\text { dose } \\
\left(\mathrm{mg} / \mathrm{m}^{2}\right)\end{array}$ & $\begin{array}{c}\text { Dose } \\
\text { intensity } \\
\text { (dose/week) }\end{array}$ & $(\%)$ & $\begin{array}{c}\text { Total dose } \\
\left(\mathrm{mg} / \mathrm{m}^{2}\right)\end{array}$ & $\begin{array}{c}\text { Dose } \\
\text { intensity } \\
\text { (dose/week) }\end{array}$ & $(\%)$ \\
\hline Cisplatin & 400 & 33.3 & 400 & 33.3 & 100 & 400 & 33.3 & 100 \\
\hline Etoposide & 1000 & 83.3 & 1500 & 125 & 150 & 1500 & 125 & 150 \\
\hline Bleomycin $^{*}$ & $430 \mathrm{U}$ & 35.8 & $270 \mathrm{U}$ & 22.5 & 63 & $180 \mathrm{U}$ & 15 & 42 \\
\hline Paclitaxel & 350 & 29.2 & 775 & 64.6 & 221 & 600 & 50 & 171 \\
\hline Oxaliplatin & 260 & 21.7 & 0 & 0 & 0 & 0 & 0 & 0 \\
\hline Ifosfamide & 12000 & 1000 & 6000 & 500 & 50 & 12000 & 1000 & 100 \\
\hline
\end{tabular}

* Total dose in units. Comparison of planned total dose and dose intensity of agents used during 12-week intensified chemotherapy period.

\begin{tabular}{|l|c|c|c|}
\hline Table 2. Patient characteristics & $\begin{array}{c}\text { UTMD + } \\
\text { intensified chemo } \\
\text { (n=6) }\end{array}$ & $\begin{array}{c}\text { UTMD + } \\
\text { standard BEP } \\
\text { (n=2) }\end{array}$ & $\begin{array}{c}\text { Favorable TMD } \\
\text { + standard BEP } \\
\text { (n=2) }\end{array}$ \\
\hline Age (years) & $30(20-51)$ & 21,32 & 18,23 \\
\hline HCG $>50000 \mathrm{IU} / \mathrm{L}$ & 1 & 1 & 0 \\
\hline AFP $>10000 \mathrm{ng} / \mathrm{mL}$ & 4 & 0 & 0 \\
\hline LDH $>10$ x ULN & 0 & 1 & 0 \\
\hline $\begin{array}{l}\text { Extrapulmonary visceral } \\
\text { metastases }\end{array}$ & 4 & 0 & 0 \\
\hline Primary mediastinal NSGCT & 0 & 0 & 1 \\
\hline ECOG performance status & 1 & 0 & 1 \\
\hline 0 & 4 & 2 & 0 \\
\hline 1 & 1 & 0 & 0 \\
\hline $2-4$ & & & \\
\hline
\end{tabular}

Data are median (range). UTMD + intensified chemo=patients with an unfavorable marker decline who received a dose-dense regimen. UTMD + Std BEP=patient(s) who received standard BEP prior to availability of GETUG-13 results. TMD inevaluable + Std BEP=patients who did not have assessable tumor marker levels but met criteria for poor prognosis disease by virtue of extrapulmonary visceral metastases. AFP: serum alfa-fetoprotein; ECOG: Eastern Cooperative 
Oncology Group; HCG: human chorionic gonadotropin; IGCCCG: International Germ Cell Cancer Collaborative Group; LDH: serum lactate dehydrogenase; NSGCT: non-seminomatous germ cell tumor; TMD: tumor markers decline; ULN: upper limit of normal; UTMD: unfavorable tumor marker decline.

Table 3. Adverse events.

\begin{tabular}{|l|l|l|l|l|l|l|}
\hline & \multicolumn{2}{|c|}{$\begin{array}{l}\text { UTMD + intensified chemo } \\
\text { (n=6) }\end{array}$} & \multicolumn{2}{l|}{ Standard chemotherapy (n=4) } \\
\hline Toxicity grade & $\begin{array}{c}\text { Grade } \\
1-2\end{array}$ & Grade 3 & Grade 4 & $\begin{array}{c}\text { Grade } \\
1-2\end{array}$ & Grade 3 & Grade 4 \\
\hline Nausea or vomiting & 4 & 0 & 0 & 2 & 1 & 0 \\
\hline Fatigue & 4 & 0 & 0 & 2 & 1 & 0 \\
\hline Diarrhea & 5 & 0 & 0 & 0 & 0 & 0 \\
\hline Liver & 4 & 2 & 0 & 1 & 1 & 0 \\
\hline Sensory neuropathy & 2 & 2 & 0 & 0 & 0 & 0 \\
\hline Dyspnea & 4 & 0 & 1 & 2 & 1 & 0 \\
\hline Hemoglobin & 1 & 4 & 1 & 2 & 2 & 0 \\
\hline Neutropenia & 3 & 0 & 3 & 0 & 0 & 1 \\
\hline Thrombocytopenia & 4 & 1 & 1 & 3 & 0 & 1 \\
\hline Febrile neutropenia & 0 & 3 & 0 & 0 & 0 & 0 \\
\hline Infection & 0 & 1 & 0 & 0 & 0 & 0 \\
\hline Deep vein thrombus & 0 & 2 & 0 & 0 & 0 & 0 \\
\hline
\end{tabular}

UTMD + intensified chemo=patients with an unfavorable marker decline who received a dose-dense regimen. Toxicity grade according to CTCAE 4.03. Infection=infectious event without neutropenic fever. UTMD: unfavorable tumor marker decline. 